\title{
Epidermal Cyst of the Plantar Foot: A Case Study
}

\author{
Charles B Parks*, Sandy Nguyen, Anmy Vu and Yen Tran \\ Department of Orthopaedic Surgery, USA
}

*Corresponding author: Charles B Parks, Department of Orthopaedic Surgery, University of California, San Francisco Orthopaedic Trauma Institute, Zuckerberg San Francisco General Hospital and Trauma Center San Francisco, CA, USA.

Received Date: January 25, 2019

Published Date: February 11, 2019

\section{Abstract \\ Epidermal inclusion cysts are benign lesions that are rarely seen on the foot due to its lack of sebaceous glands. (Paparelli) We report a case of a 70-year-old male with an inclusion cyst for over 5 years. Radiographs and magnetic resonance imaging revealed the presence of subcutaneous mass with a growing epidermoid.}

Keywords: Epidermal inclusion cyst; Epidermoid cyst; Infundibular cyst

\section{Introduction}

Epidermal inclusion cysts (also commonly referred to as epidermal cysts, epidermoid cysts and infundibular cysts) are benign cysts in the epidermal region of the skin. Comprising about $85-90 \%$ of all excised subcutaneous cysts, they are relatively common. (Kim, Nigam) The cyst is most often found in the hair bearing surfaces of the body due to a displacement and proliferation of the epidermal cells within the dermis, or from inflammation of the pilosebaceous glands (Figure 1).

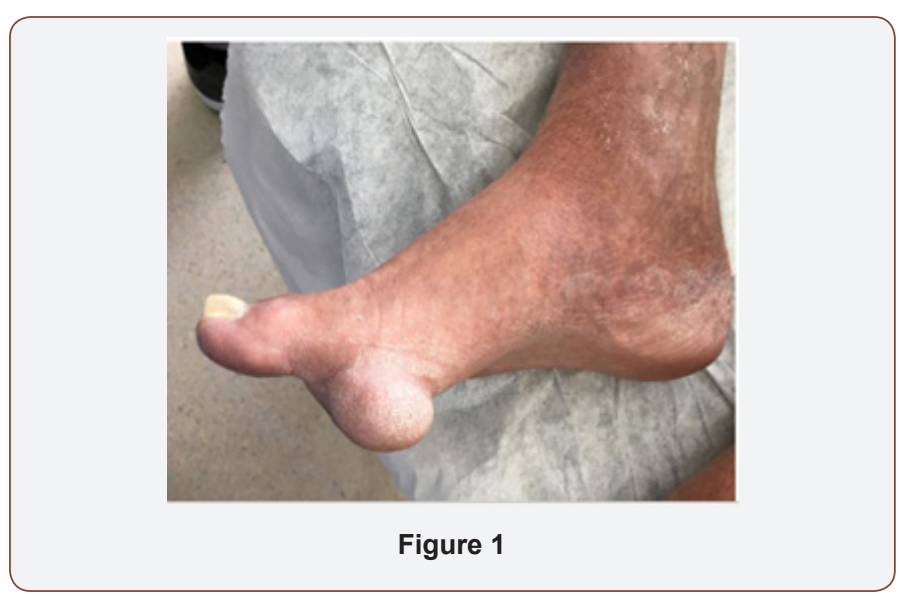

The most common locations include the head, neck, trunk, extremities, with less than $10 \%$ presenting in the lower extremity. (Nigam, Coulter) However, epidermoid cysts may occasionally appear on non-hair-bearing surfaces of the body. These cysts are thought to develop due to a history of trauma or surgery that implanted the epidermal cells in the area. Specifically, the cysts develop from epidermal fragments into a heterotrophic location. The cyst enlarges through keratinous debris and epithelial accumulation, resulting in a subcutaneous mass (Figure 2) [1].

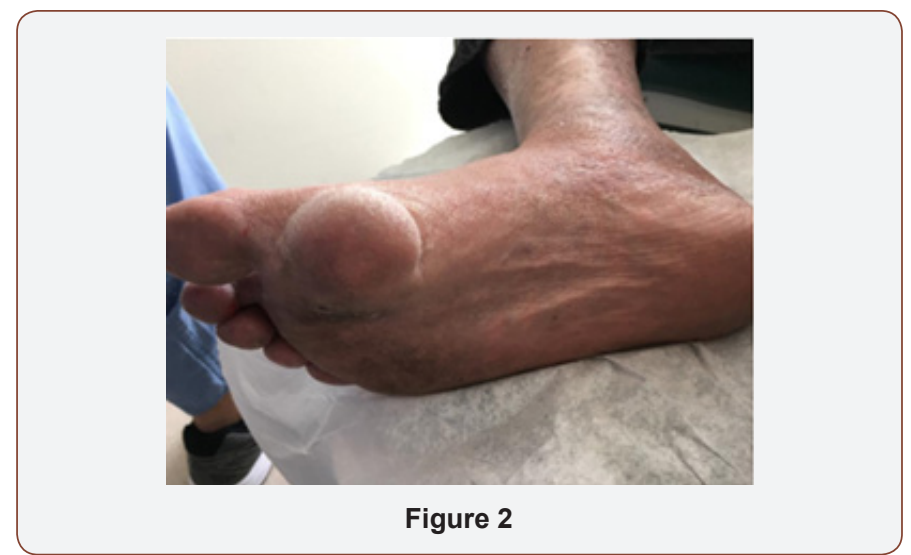

In the foot, epidermoid cysts occurred most commonly in the $1 \mathrm{st}$ toe, followed by the ankle, 2 nd toe, 3 rd toe, 4 th toe, 5 th toe, and heel. The average size of the cyst in the foot is $1.6 \times 1.5 \times 0.8 \mathrm{~cm}$. Treatment of epidermoid cysts include excision but recurrence rates appear to be high at $18.9 \%$ [2] Epidermal inclusion cysts present clinically as well-encapsulated, freely movable, elevated subcutaneous masses. The masses contain a yellow and white substance in the center. [1] Since 2000, there has only been 2 reported cases of epidermoid cysts on the plantar foot. (Ramak, Basterzi) We will be reporting a 3rd case within a period of 17 years. 


\section{Case Report}

Our patient, a 70 year old with past medical history significant for diabetes mellitus type II, peripheral neuropathy, myocardial infarction, congestive heart failure, hypertension, obesity and hypercholesterolemia presented to our clinic complaining of a painless, soft tissue mass under his right big toe that has been present for over 5 years. The mass has enlarged over the years causing stress on the lateral aspect of his foot and ankle due to gait compensation. Patient notes he had a previous ulceration at this location for 3 years. He noticed the mass beginning to form once the ulceration had completely healed.

On clinical examination, the mass measured at $6.0 \mathrm{~cm} \times 7.0$ $\mathrm{cm} \times 2.0 \mathrm{~cm}$ on the sole of the foot (Figure 1\&2). Lesion was wellcircumscribed with no tenderness to palpation. The elevated lesion had a rubbery texture to it and was adherent to the skin (Figure 3).
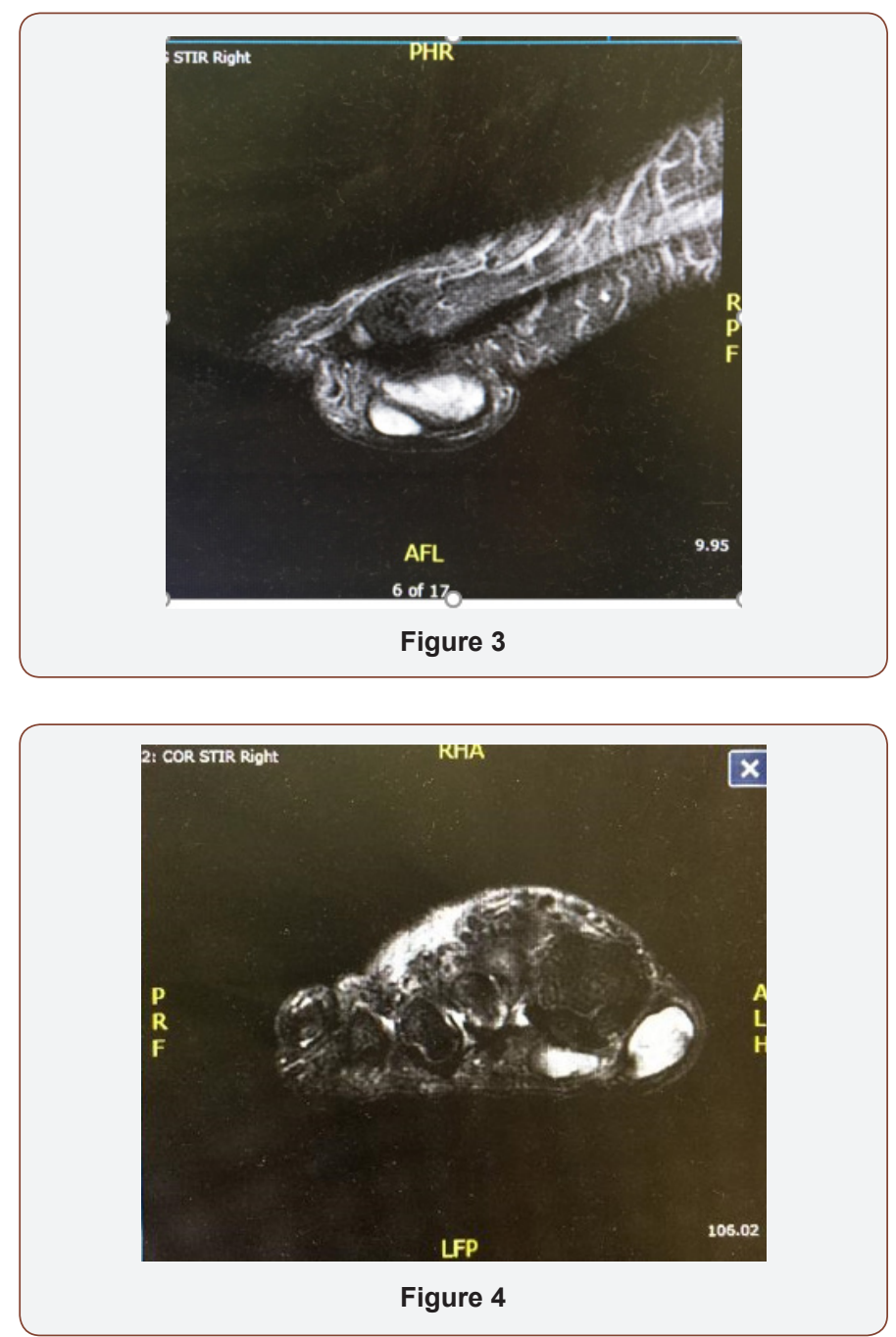

Patient had radiographs and magnetic resonance imaging (MRI) obtained prior to his visit and preoperatively. MRI findings in June 2016 prior to visit revealed two adjacent lobular well-defined T2 and STIR heterogeneously hyperintense and T1 hyperintense lesions in the plantar superficial soft tissues immediately adjacent to the first metatarsophalangeal joint. Both portions of the lesion were slightly increased in size compared to prior imaging in November 2013 with the more medial lesion now measuring $1.8 \mathrm{~cm} \times 2.9 \mathrm{~cm}$ x $1.7 \mathrm{~cm}$ (previously $2.0 \mathrm{~cm}$ x $2.5 \mathrm{~cm} \mathrm{x1.0} \mathrm{cm)} \mathrm{and} \mathrm{the} \mathrm{more} \mathrm{lateral}$ septated lesion measuring $2.1 \mathrm{~cm} \times 1.8 \mathrm{~cm} 1.1 \mathrm{~cm}$ (previously 1.4 $\mathrm{cm} \times 1.0 \mathrm{~cm} \times 0.7 \mathrm{~cm}$ ), approximately $4.1 \mathrm{~cm} \times 2.8 \mathrm{~cm} \times 1.2 \mathrm{c} . \mathrm{m}$ in aggregate, which may represent fibroma, foreign body reaction or proteinaceous cyst (Figure 4). There were no involvement of the joint space, flexor hallucis tendon or first metatarsal, phalanges or sesamoids. Given the slow interval growth, malignancy was considered less likely. Radiographs prior to visit revealed interval increased size of mass along the plantar foot with no evidence of adjacent osseous erosion within the first metatarsal, proximal phalanx or distal phalanx (Figures 3-5) [3].

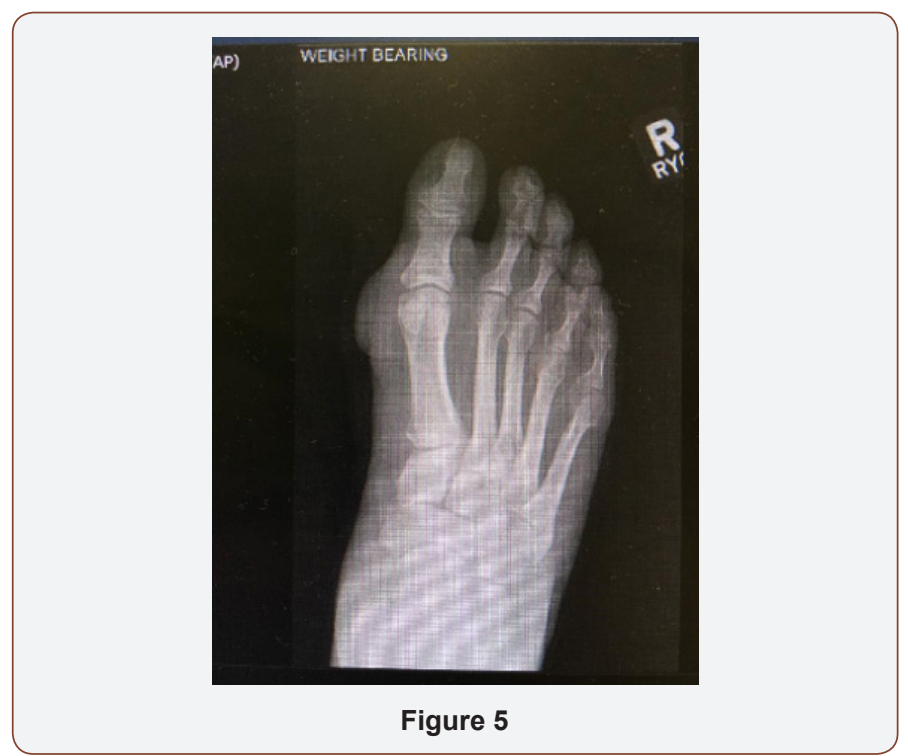

A popliteal saphenous block was performed by the anesthesiologist prior to entering the operating room. The patient was then brought to the operating room and placed on the table in a supine position and sedated with MAC. An ankle tourniquet was applied to the extremity and inflated to $250 \mathrm{mmHg}$. Attention was then directed to the right foot where there was a large 3.0 $x 3.0 \mathrm{~cm}$ mass on the plantar medial aspect of the right first metatarsophalangeal joint. Using sharp and blunt dissection, the incision was deepened with care taken to identify and avoid or cauterize any bleeders. The incision was deepened using a combination of sharp and blunt dissection and the most dorsal portion of the soft tissue mass was identified. Blunt dissection was continued until 2 pedicles were identified and resected at their most proximal areas. The multi-lobulated $3 \mathrm{~cm}$ soft tissue mass was bi-pedunculated, well adhered to the plantar soft tissue metatarsal fat pad, with no involvement with any surrounding bony structures or soft tissue structures, absence of tortuous vessels or nerves within the soft tissue mass. The skin edges were re-approximated using \#3-0 nylon and simple interrupted suture with adequate eversion of skin edges. The wound was then dressed in betadine soaked adaptic, gauze, kerlix and coban.

At 1 week postoperatively, the patient was seen at our clinic. The surgical site was examined and we reviewed pathology results with the patient. The pathology findings confirmed an epidermal inclusion cyst. Patient continued to apply xeroform and dry sterile dressing with coban to right foot and to continue weightbear in a post-op shoe. At week 2, sutures were removed and was 
cleared to weight-bear in tennis shoes. At this time, radiographs were taken which revealed interval removal of the previously described soft tissue mass along the plantar aspect of the right first metatarsophalangeal joint with soft tissue swelling at the plantar surface of the forefoot and medial first metatarsal soft tissues. At 6-month follow-up, patient was doing well and no complaints of pain at the incision site (Figure $6 \& 7$ ) [4-7].
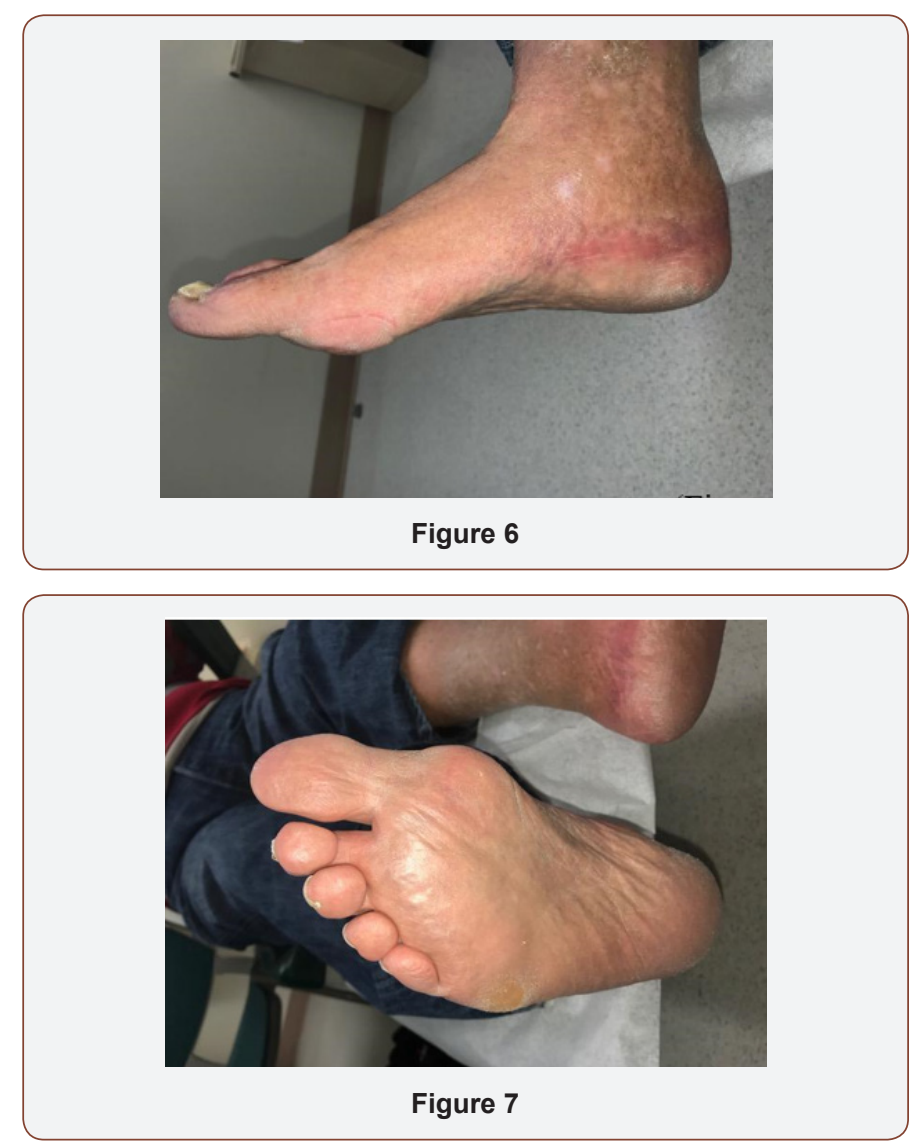

\section{Discussion}

Epidermal inclusion cysts are the most commonly excised subcutaneous cysts, but are present less than $10 \%$ of the time on the hair-bearing surfaces of the extremities. Thus, it is extremely rare to see epidermal inclusion cysts on the non-hair bearing palms and soles. This case study highlights the third case of an epidermal inclusion cyst seen in the foot.

The etiology of these lesions can be due to embryonically misplaced epidermoid tissue, implantation of hair, matrix or a foreign body below the epidermis or obstructed or occluded follicles. (Coulter) It is interesting to note that the patient had a history of ulceration at the site of the subsequent epidermal inclusion cyst, a history which may have induced its growth. Therefore, a proper history from the patient may direct the focus to several dermatological differentials which may include epidermal inclusion cysts on the plantar hallux, although rare.

\section{Conclusion}

Epidermal inclusion cysts are benign lesions that respond well to total excision, but they are rarely seen on the non-hair bearing surfaces of the body. As reported with our case study, it is important to perform a thorough history and physical so that your differential diagnosis's are adequate. When seeing a lesion in the foot, epidermal inclusion cysts should be on the differential and one should always ask if there was any history of trauma, surgery or open wounds in the area. Epidermal inclusion cysts are benign lesions which respond well to excision of the lesion as treatment.

\section{Acknowledgement}

None

\section{Conflicts of interest}

No conflict of interest.

\section{References}

1. Nigam JS, Bharti JN, Nair V, Gargade CB, Deshpande AH, et al. (2017) Epidermal cysts: A clinicopathological analysis with emphasis on unusual findings. International journal of trichology 9(3): 108-112.

2. Young KW, Kim JS, Joh JW, Yeom CH, Park YU et al. (2013) Epidermal Cyst in the Foot and Ankle. Journal of the Korean Orthopaedic Association 48(2): 104-110.

3. Ramakrishnaiah SB, Rajput SS, Gopinathan NS (2016) Epidermoid Cyst of the Sole-A Case Report. Journal of clinical and diagnostic research 10(11): PD06-pd07.

4. Basterzi Y, Sari A, Ayhan S (2002) Giant epidermoid cyst on the forefoot. Dermatologic surgery 28(7): 639-640.

5. Coulter P, Bouche R (1999) Traumatically induced inclusion cyst: Secondary to shoe impingement: Report of three cases. J Foot Ankle Surg 38(4): 271-277.

6. Kim H, Kim S, Lee S, Racadio J, Shin M (2011) Subcutaneous epidermal inclusion cysts: Ultrasound and MR imaging findings. Skeletal Radiology 40 (11): 1415-1419.

7. Paparella T, Fallat L (2018) A Rare Presentation of a Giant Epidermoid Inclusion Cyst Mimicking Malignancy. J Foot Ankle Surg 57(2): 421-426. 